\title{
Price and Profitability From Two-Quality Retread Tire
}

\author{
Jumpol Vorasayan \\ Department of Agro-industrial Technology, Faculty of Agro-Industry \\ Kasetsart University, Bangkok, Thailand.
}

\begin{abstract}
Tire manufacturers such as Michelin and Goodyear sell their new and retread tire on their stores. Alternatively, there are independent dealers that also sell the similar retread product via their own channels. In a two-player game theory, increasing one's profit would result in decreasing the other. This research demonstrates how to find profits by changing price position of retread tire from an OEM and the 3rd party dealer. Profit function bases on price and relative perceived quality to the new product. The simulation results show that mutual profit of both parties is improved by introducing retread tire, however, price competition from individual profit maximization prevents both parties to set the price point that maximize their mutual profits.
\end{abstract}

Keywords: Market Segmentation, Price Analysis, Remanufactured Products, Retread Tire, Rev erse Supply Chain.

\section{INTRODUCTION}

The lifespan of tire is longer than the period that the first owner replace it. The used tire can be repaired and reused for budget tra nsportation. Additionally, wear off tire could be retread by replacing the old rubber layer wi th new one. Retread tire off a reasonable alter native to the new one and it is environmental friendly because this method can save up to 8 $0 \%$ of the tire material cost (Debo and Van W assenhove [1]). A tire can be retread up to five times. We can foresee a tremendous number of used products in the market. Similarly, in $\mathrm{f}$ ood industry that product value decrease over time, price of nearly expired product must be adjusted in order to attract customer and be so ld before losing its value.

In this research, we demonstrate how to set a price of two different type of retread $p$ roduct from two main used-product sellers in the market, an original equipment manufactur er (OEM) and a dealer. We name retread prod ucts which are sold by original owners via co mmon dealer as general used products (GRP). On the other hand, the other portion of used $p$ roducts from consumer or product rental com panies are purchased by the OEM then are ins pected, repaired and tested by OEMs standard until the quality of meets the standard. We cal l these certified retread products (CRP). Exam ples of OEM that resell their retread products are Michelin and Goodyear while examples of the 3rd party company who sell their retread $t$ ire are kaltire.com and tirerecappers.com. The se two players compete in the retread tire mar ket by the quality and price differentiation. In line with Vorasayan and Ryan [2], we use a w ord "perceived quality" to represent i.e. performance, warranty period, seller's reputat ion. The actual performance of each retread pr oduct can be varied and cannot be evaluated $b$ y historical information such as production da te or physical appearance. Average consumer without in-depth knowledge on rubber and tir e could not tell the performance differences b y just having a short test drive. Therefore, war ranty and seller reputation are key factors in c ustomer confidence in retread product. CRP c ome with technology and warrantee from the OEM. Consumer, thus, perceive that it has hig her quality than typical retread products and a re willing to buy it with higher price. Profit $m$ argin and quantity of sales are surviving facto rs. In general, both parties are not cooperative and want to maximize their own profits. High er profit for one party could results in lesser $p$ rofit from the other. How can we find the pric e that satisfies both parties in this two-player game? How much total profit would be impro ved if both parties cooperate and agree to shar

ISSN 2413-0877 (C) 2016 The Authors.

Published by KnowledgeE Publishing Services. This is an open access article under the CC BY-NC-ND license (http://creativecommons.org/licenses/by-nc-nd/4.0) 
e a fair proportion of mutual profit?

Studying resells of remanufactured go ods can be categorized under the market segm entation in closed-loop supply chain where ite $\mathrm{m}$ has been redistributed to consumer more th an one time. It received great attention in the past 10 years due to cost saving and natural $\mathrm{c}$ onservation (Drake and Furguson, [3]). The st udy by Hauser and Lund [4] explained that pri ce differentiation of remanufactured products come from the variations in warranties, produ ct inspection points, and other program comp onents. This incidence implies that customer $n$ eeds to choose the program, hence, price that ally with his/her expectation. Debo [5] and Jin et. al. [6] presents a consumer profile functio $\mathrm{n}$ for new and remanufactured products. Subra manyam and Subramanyam [7] studies motiv ation and satisfaction of buyers after buying $\mathrm{p}$ re-owned products from ebay.com. They foun $\mathrm{d}$ that satisfaction from buying pre-owned pro ducts does not necessarily lower than from bu ying new products. The study also states price difference between new and pre-owned prod ucts based largely on seller reputation and war ranty period. Oraiopoulos [8] shows that $77 \%$ of information technology companies buy pre -owned products and $46 \%$ of these companies continually increase their budgets for pre-ow ned products in the next fiscal year.

In this paper, we develop a mathematical model for multiple qualities of products 1) new, 2) certified retread with warrantee by the OEM, and 3) noncertified retread tire by 3rd parties. The model consists of price, quality, cost and demand to give an insight of how the optimal price position under cooperative and non-cooperative schemes.

\section{METHOD}

The valuation of consumer in the market $(f(x))$ is assumed to be uniformly distributed with parameter $[a, b]$ i.e. if we sort consumers from their purchasing power, the difference in purchasing power is constant. $a$ and $b$ are minimum and maximum price for used product in the market. When $P_{n} \leq a$, every prospect consumer in the market will buy new product. In contrast, when $P_{n}>b$, all consumer will not buy it. When simplify $[a, b]$ to [0,1]. At $P_{n}=0.3$, it implies that $70 \%$ of prospect consumers in the market have valuation more than $P_{n}$ and will buy new products if there is no other alternative. Other notations are

$P_{n} \quad=$ price of a new tire

$P_{u 1} \quad=$ price of a certified retread tire

$P_{u 2} \quad=$ price of a noncertified retread tire

$\lambda_{n} \quad=$ demand for new tire

$\lambda_{u 1}=$ demand for certified retread tire

$\lambda_{u 2}=$ demand for noncertified retread tire

$\delta_{1}=$ perceived quality of certified retread tire

$\delta_{2}=$ perceived quality of noncertified retread tire

$v \quad=$ valuation of a consumer,

$c_{n} \quad=$ cost of a new tire

$c_{u 1}=$ cost of a certified retread tire

$c_{u 2}=$ cost of a noncertified retread tire

Let quality of a brand-new tire equals to 1. $\delta_{1}$ and $\delta_{2}$ are quality of certified and noncertified products are respectively where $0<\delta_{2}<\delta_{1}<1$. Valuation is assumed to be direct proportional to perceived quality. For example if valuation for a new product is $v$ then valuation for certified and noncertified products are $\delta_{1} v$ and $\delta_{2} v$ respectively.

Demand function can be derived by these logical expressions.

1. Consumer will buy a new product if

$1.1 v-P_{n}>0$ and

$1.2 v-P_{n}>\delta_{1} v-P_{u 1}$ and

$1.3 v-P_{n}>\delta_{2} v-P_{u 2}$

or $v \geq \max \left\{P_{n}, \frac{P_{n}-P_{u 1}}{1-\delta_{1}}, \frac{P_{n}-P_{u 2}}{1-\delta_{2}}\right\}$

For $\lambda_{n} \geq 0$,

$\max \left\{P_{n}, \frac{P_{n}-P_{u 1}}{1-\delta_{1}}, \frac{P_{n}-P_{u 2}}{1-\delta_{2}}\right\} \leq 1$

and,

$\lambda_{n}=\int_{\max \left\{P_{n}, \frac{P_{n}-P_{u 1}}{1-\delta_{1}}, \frac{P_{n}-P_{u 2}}{1-\delta_{2}}\right\}}^{b} f(x) d x$.

Then, maximum prices of retread products are

and

$$
P_{n}-b\left(1-\delta_{1}\right)>P_{u 1}
$$

$$
P_{n}-b\left(1-\delta_{2}\right)>P_{u 2}
$$


2. Consumer will buy a certified retread product if

$2.1 \delta_{1} v-P_{u 1}>0$ and

$2.2 \delta_{1} v-P_{u 1}>v-P_{n}$ and

$2.3 \delta_{1} v-P_{u 1}>\delta_{2} v-P_{u 2}$

or $\max \left\{\frac{P_{u 1}}{\delta_{1}}, \frac{P_{u 1}-P_{u 2}}{\delta_{1}-\delta_{2}}\right\} \leq \lambda_{u 1}<\frac{P_{n}-P_{u 1}}{1-\delta_{1}}$

For $\lambda_{u 1}>0, \max \left\{\frac{P_{u 1}}{\delta_{1}}, \frac{P_{u 1}-P_{u 2}}{\delta_{1}-\delta_{2}}\right\} \leq 1$

therefore,

$\lambda_{u 1}=\int_{\max \left\{\frac{P_{u 1}}{\delta_{1}}, \frac{P_{u 1}-P_{u 2}}{\delta_{1}-\delta_{2}}\right\}}^{\frac{1-\delta_{1}}{1}} f(x) d x$

and,

$$
P_{u 1}<\delta_{1} P_{n}
$$

3. Consumer will buy a noncertified retread product if

$3.1 \delta_{2} v-P_{u 2}>0$

$3.2 \delta_{2} v-P_{u 2}>v-P_{n}$

$3.3 \delta_{2} v-P_{u 2}>\delta_{1} v-P_{u 1}$

or $\frac{P_{u 2}}{\delta_{2}} \leq \lambda_{u 2}<\min \left\{\frac{P_{n}-P_{u 2}}{1-\delta_{2}}, \frac{P_{u 1}-P_{u 2}}{\delta_{1}-\delta_{2}}\right\}$

therefore,

$\lambda_{u 2}=\int_{\frac{P_{u 2}}{\delta_{2}}}^{\min \left\{\frac{P_{n}-P_{u 2}}{1-\delta_{2}}, \frac{P_{u 1}-P_{u 2}}{\delta_{1}-\delta_{2}}\right\}} f(x) d x$.

In order to have $\lambda_{u 2}>0$,

$$
P_{u 2}<\delta_{2} P_{n}
$$

For $i$ type of a product sold, assuming all demands are met, profit is $\lambda_{i}\left(P_{i}-c_{i}\right)$. We find the optimal prices of retread tires to maximize profit under 3 schemes.

1) Profit for an individual party with no competition.

2) Mutual profit of OEM and the 3rd party dealer.

3) Mutual profit of OEM and the 3rd party dealer with competition.

In the situation that there are demands and profits for all types of products, minimum and maximum values of variables $P_{u 1}, P_{u 2}$ are

$\operatorname{Max}\left(C_{u i}, P_{n}-\left(1-\delta_{i}\right)\right) \leq P_{u i} \leq \delta P_{n}$,

$i=1,2$

and

$$
P_{n} \delta_{1} \geq P_{u 1} \geq \frac{\delta_{1} P_{u 2}}{\delta_{2}}
$$

\section{RESULTS}

We simulated perceived quality of retread product so than the average perceived quality of certified product is $80.1 \%$ of the brand new product. The average price for a noncertified retread tire is $72.8 \%$. Hence, we set the $\delta_{1}=0.84$ and $\delta_{2}=0.8$.

Price of a brand-new product $\left(P_{n}\right)$ is set to 0.5 . At this price, $50 \%$ of consumers see value of the product more than its price so they would buy it if there is no other alternative. Cost parameters are adjusted proportional to $P_{n}$ however real cost of all parties are still unclear since cost of purchasing retread tire can be varied depending on consumer's willingness to sell, negotiation skills and market situations. Let our initial cost parameters be $c_{n}=0.40, c_{u 1}=$ 0.25 and $c_{u 2}=0.21$. This is the case where gross margin from selling new is in the middle between maximum and minimum profit margin from selling retread tire, specifically $\operatorname{Min}\left(P_{u 2}-c_{u 2}\right)<\operatorname{Min}\left(P_{u 1}-\right.$ $\left.c_{u 1}\right)<P_{n}-c_{n}<\operatorname{Max}\left(P_{u 1}-c_{u 1}\right)<$ $\operatorname{Max}\left(P_{u 2}-c_{u 2}\right)$.

We first shows the optimal $P_{u 1}$ for OEM where noncertified product is not present. In Figure. 1, we can see there are 2 local optima separated by a line indicating demands for new products. Profits on the left are from $\lambda_{u 1}$ only while profit on the right comes from demands from both kinds of products. The optimal $P_{u 1}$ is 0.377 . For instance, a brand new product cost 20,000 THB therefore the optimal price of certified retread tire in this case is $\frac{20,000}{0.5} 0.377=15,080 \mathrm{THB}$ 


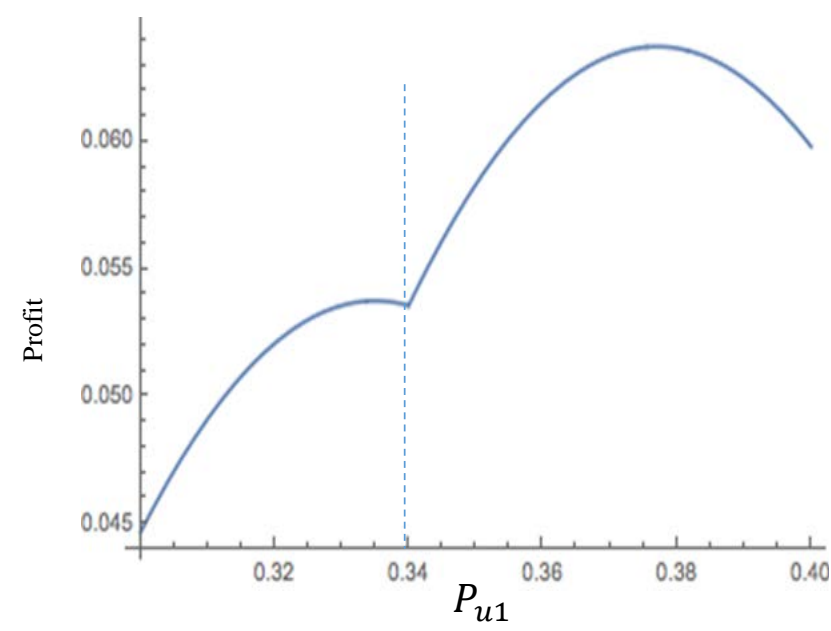

Figure. 1 Profit of OEM from selling new and certified products in no the 3rd party dealer scheme

In the case with no certified products, profit of the dealer as concave respect to the price. The price that maximize noncertified products is $\mathrm{P} \_\mathrm{u} 2=0.2975$ or $11,900 \mathrm{THB}$ as shown in Figure 2.

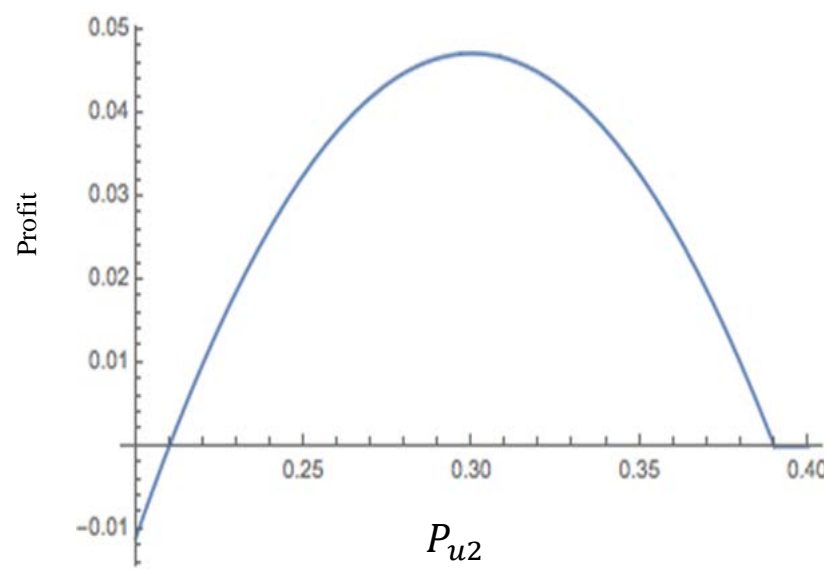

Figure. 2 Profit of the dealer from selling noncertified products under in no certified products scheme

For 2 variables, the mutual profit is presented by contour plot of profit as a function of $P_{u 1}$ and $P_{u 2}$ as in Figure 3. The vertical and horizontal white lines are $\frac{P_{n}-P_{u 1}}{1-\delta_{1}}=1$ and $\frac{P_{n}-P_{u 2}}{1-\delta_{2}}=1$ respectively. The area inside these lines is where $\lambda_{n}>0$. The brighter tone indicates the more profit. The upper diagonal white line is where $\frac{P_{u 1}}{\delta_{1}}=\frac{P_{u 2}}{\delta_{2}}$. The lower diagonal line is where $\frac{P_{n}-P_{u 1}}{1-\delta_{1}}=$ $\frac{P_{n}-P_{u 2}}{1-\delta_{2}}$. The area on the left of upper diagonal is where there is no demand for noncertified while the area on the right of lower diagonal is where there is no demand for certified products. Consequently, there are demands for both in the middle area.

When both parties are cooperating, we can see 2 local optima. One (point Y) is in an area where no demand for new product and the other one is in the area with demands for both new and retread products (point $\mathrm{X}$ ). At point $\mathrm{X}$, the maximum mutual total profits if OEM and the $3^{\text {rd }}$ party dealer are co-operated are achieved by setting $P_{u 1}=0.377$ or 15,080 THB and $P_{u 2}=0.336$ or 13,440 THB. It is in the area where demands for new and both kinds of retread products are presented. At this point, total demands for all products increased to $56.36 \%$ compared with 50\% when there are no retread tire in the market. The market shares for new, certified and noncertified retread tire are $41 \%, 32 \%$ and $27 \%$ or $23 \%, 18 \%$ and $15 \%$ of market size respectively.

Figure 4 and 5 shows 2 distinct contour graphs of profit from OEM and the 3rd party dealer. As in Figure. 1 and 2, OEM's profit is maximized when $P_{u 1}=$ 0.377 (Point A). The 3rd party dealer's profit is maximized when $P_{u 2}=0.2975$ (Point $\mathrm{B}$ ). We can see that the optimal point of each party is not in the same region thus it cannot be mutually optimized. When these 2 parties are not cooperating, the optimal prices can be situational. For example, the optimal point in cooperative case (point $\mathrm{X}$ ) is not equilibrium point. Either party will get profit boost by lowering his product price. Combined with reason such as excess inventory. $3^{\text {rd }}$ party dealer decided to lower $P_{u 2}$ down to raise demand to match his supplies and increase the profit. He lowers $P_{u 2}$ down to 0.33 or 13,200 THB. At this point, the demand for $3^{\text {rd }}$ party retread tire raised from $15 \%$ to $24 \%$ of the market. On the other hand, because of the price reduction, demand of recertified product declined from 18\% to 9\%. OEM, supposedly, with retread products supply of $18 \%$ of the market, is enforced to lower $P_{u 1}$ down to regain his market share. However, he has to be cautious about reducing $P_{u 1}$ because it will again negatively affect $\lambda_{u 2}$ that will leads to another reduction in prices. If price reduction kept going, it would lead to the point where 
all parties has zero profit. So, the optimal price should not be at the extreme points where demands of either retread product is zero. Both parties have to find the acceptable balance between inventory cost and profit margin in order to maintain reasonable profits. In this particular case, OEM might decide to lower price down only slightly to gain some of his demand for retread tire back. At point $\mathrm{D}, P_{u 1}=0.375$ or 15,000 THB and $P_{u 2}=0.330$ or 13,320 THB that leads to reasonable demands for both retread products are $\lambda_{u 1}=14 \%$ and $\lambda_{u 2}=21 \%$. At this point, the OEM lose profit by $4.25 \%$ while $3^{\text {rd }}$ party dealer gain profit by $9.32 \%$. The equilibrium prices of both retread tire can be found in Point $\mathrm{F}$ but it is unlikely to reach the point where both parties lose 50\% of profits from point $\mathrm{X}$ and OEM sell only retread tires.

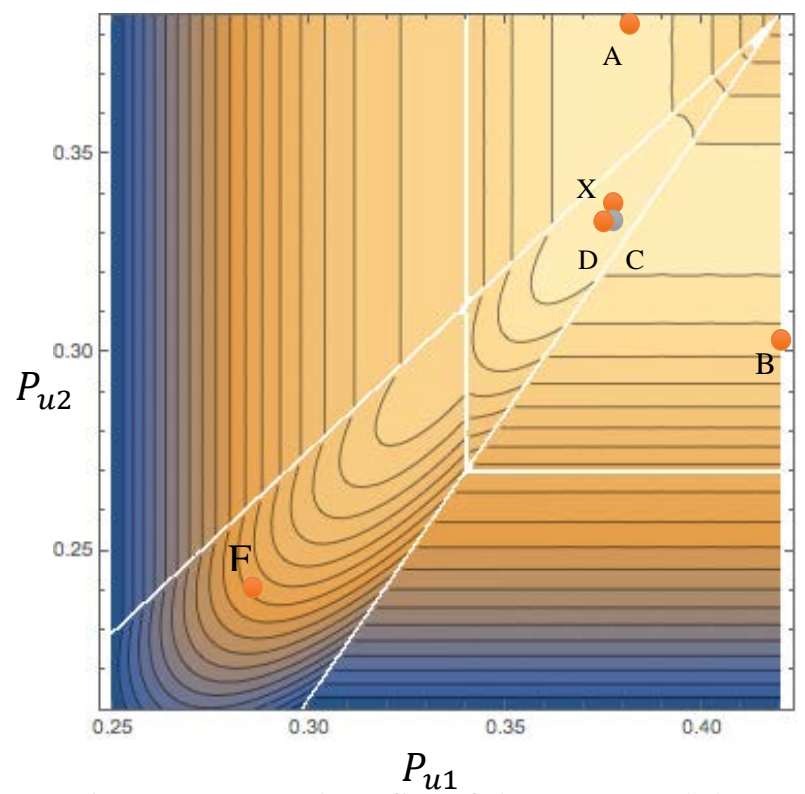

Figure. 3 Mutual profits of the OEM and the 3rd party dealer from selling new and retread tire

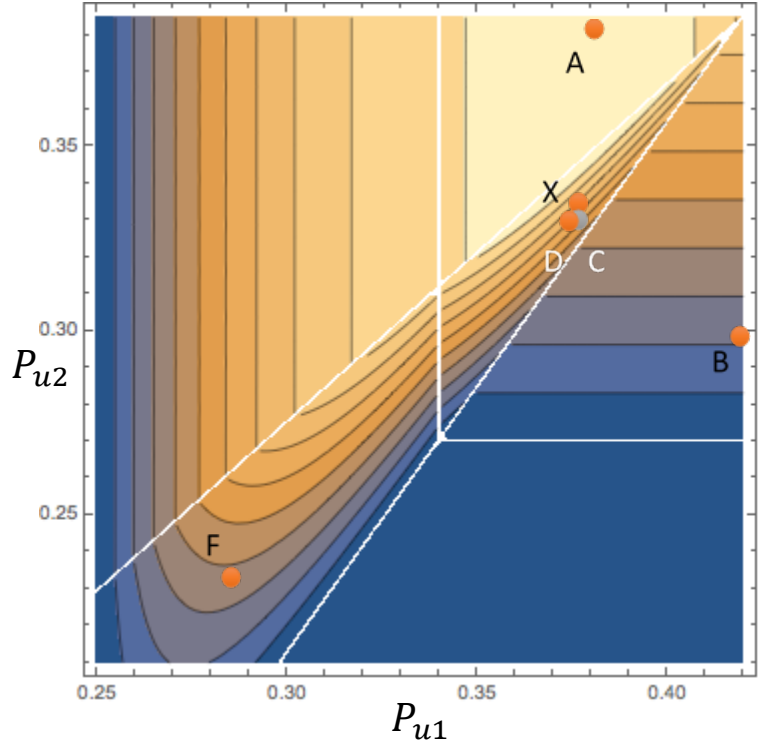

Figure. 2 Profits of the OEM from selling new and certified pre-owned tires

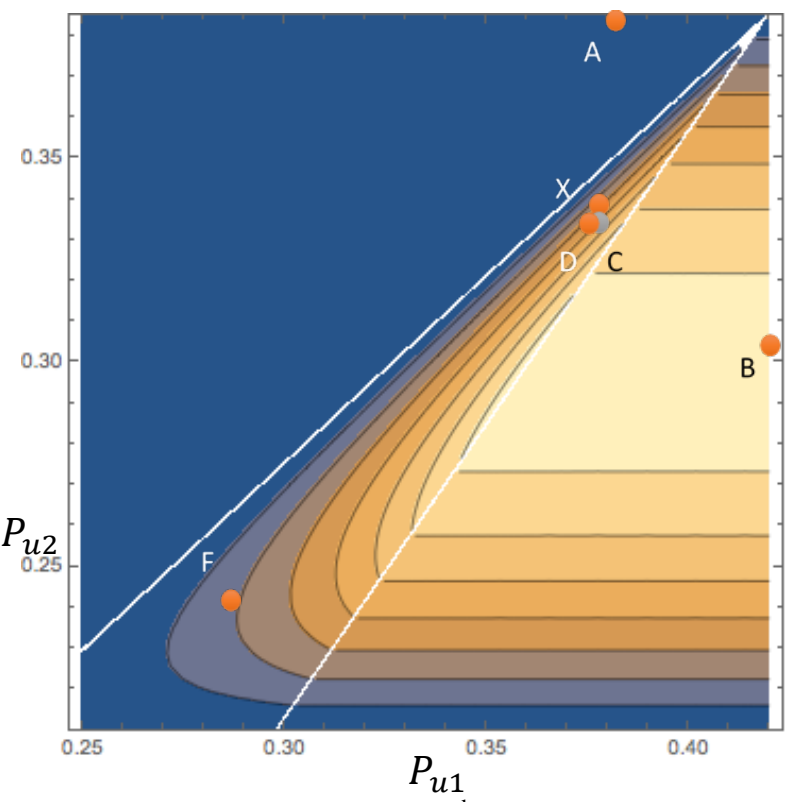

Figure. 3 Profit of the $3^{\text {rd }}$ party dealer.

Profits in Fig. 3, 4 and 5 base on estimate cost which might not be true. The less cost, the more possible profit and it would lead to lower optimal prices. The model itself still needs modification in order to find applicable optimal points. In practice, the uniform purchasing power is rather not a good representative of the market. The distribution of income might have positive skewed shape because the number of high 
income buyers is less than low income buyers. Factors such as store brand, store location, affect buyer perceived qualities and lead to different prices. On the reseller side, holding cost of used product, inventory level, the release date of coming new model, new product backorders are also related to product prices. The optimal prices are also related to the assigned values of quality of used products. In example, they are relatively high due to assumption in demand function. That probability distribution of qualities could be subject for future study.

\section{CONCLUSION}

We develop a mathematical model to determine prices of used products sold from 2 parties, OEM and the 3rd party dealer. The retread tire from OEM has higher perceived quality because of brand reputation and loyalty of customer. Noncertified product from the 3rd party dealer, on the other hand, competes with lower price. Under cooperative scheme, local optimal prices could be found numerically. The global optimum and equilibrium were found heuristically. In noncooperative scheme, maximize profit in one party will lower profit of the other down. Both parties need to settle for satisfactory profit rather than continuously cutting prices down to retain profitable business. The market of remanufactured product is not limited to retread tire but any agricultural products that have as well as consumer and electronics products that have been refurbished or repaired to a certain quality relative to new product quality. With competitive prices, certified remanufactured products could give higher consumer surplus than other alternatives. It could leads to lower overall material consumption.

\section{ACKNOWLEDGEMENT}

This research was partially supported by the Khon Kaen University Research Fund.

\section{REFERENCES}

Debo, Laurens G., L. Beril Toktay, Luk N. Van Wanssenhove. 2005. Merket Segmentation and Product Technology Selection for Remanufacturable Products.
Management Science, 51(8): Page 1193 Page 1205.

Debo, Laurens G., and Luk N. Van Wassenhove. 2005. Tire recovery: the RetreadCo case. In Managing Closed-Loop Supply Chains. Pp.119-128. Springer Berlin Heidelberg

Drake M. J. and Ferguson M. E. 2010 Closed-Loop Supply Chain Management for Global Sustainability in Global Sustainability Initiatives: New Models and New Approaches, In Press

Hauser, W, R. T. Lund. 2003. Remanufacturing: An american perspective. Boston University, Boston.

Jin Y., Muriel A., and Y. Lu. 2011. On the Profitability of Remanufactured Products. Working paper, University of Massachusetts, Amherst.

Oraiopoulos N., Ferguson, M. E. and Toktay, B. L. 2012. Relicensing as a Secondary Market Strategy. Management Science. 58(5): page 1022-page 1037

Subramanian R. and Subramanyam R. 2012. Key Drivers in the Market for Remanufactured Products: Empirical Evidence from eBay. Manufacturing and Service Operations Management. 14(2): page 315 - page 326

Vorasayan, J. and Ryan, S. M. "Optimal Price and Quantity of Refurbished Products" Production \& Operations Management, 2006, 15(3): page 369 - page 383. 